\title{
Partially Typed Terms between Church-Style and Curry-Style
}

\author{
Ken-Etsu Fujita ${ }^{1}$ and Aleksy Schubert ${ }^{2}$ \\ 1 Shimane University, Matue 690-8504, Japan, \\ fujiken@cis.shimane-u.ac.jp \\ 2 Warsaw University, 02-097 Warsaw, Poland, \\ alx@mimuw .edu.pl
}

\begin{abstract}
We introduce several structures between Church-style and Curry-style based on partially typed terms formalism. In the uniform framework, we study the static properties of the $\lambda$-terms between the two styles. It is proved that type checking, typability, and type inference for domain-free $\lambda 2$ are in general undecidable. A simple instance of the second-order unification problem is reduced to the problem of type inference for domain-free $\lambda 2$. The typability problem is undecidable even for a predicative fragment of domain-free $\lambda 2$, called the rank 2 fragment. It is also found that making polymorphic domains free and the use of type-holes [ ] are independently responsible for the undecidability of the partial polymorphic type reconstruction problem.
\end{abstract}

\section{Introduction}

There are known three styles of (typed) $\lambda$-terms, called Curry-style, Churchstyle, and domain-free style. For some systems such as simply typed $\lambda$-calculus and ML 219, it is well-known that the Curry-style and the corresponding Church-style are essentially equivalent 1 15. Hence, the Curry system serves as a short-hand for the Church system. On the other hand, recently, Barthe, Sørensen, and Hatcliff 34 introduced the notion of domain-free pure type system. Terms in domain-free style have domain-free $\lambda$-abstraction. Barthe and Sørensen posed a question to know whether the problem of type checking is decidable for domainfree $\lambda 2$ and $\lambda \omega$ (page 18 [4]). In this paper, we will show that type checking, typability, and type inference are, in general, undecidable for domain-free $\lambda 2$. In order to prove this, we reduce simple instances of the second-order unification problem to the problem of strong type inference for domain-free $\lambda 2$.

Original motivation for domain-free systems comes from a study of classical type system which is an extension of intuitionistic type theory together with classical rules such as double negation elimination. The domain-free systems are useful to give continuation-passing style translations [3|10] which provide a certain semantics of classical type system. Further, when we construct a polymorphic call-by-value calculus with control operators such as callcc or $\mu$-operators [24], the Curry style cannot work for a consistent system. For instance, see the traditional counterexample (ML with callcc is unsound) by Harper\&Lillibridge 
[14], and see also a proof-theoretical observation in [10]. Hence, domain-free $\lambda \mu$ calculus has been introduced in [10], where the explicit type annotations for polymorphic terms play a role of choosing an appropriate computation under call-by-value. Our result in this paper also gives a negative answer to the problem of type checking for second-order $\lambda \mu$-calculus in domain-free style, which is a variant of Parigot's $\lambda \mu$-calculus in Curry style [24.

Domain-free systems are also useful for a study of partial polymorphic type reconstruction. Boehm [2] and Pfenning [25] have proven that the partial type reconstruction problem is, in general, undecidable for second-order $\lambda$-calculus. The typability problem for domain-free $\lambda 2$ can be regarded as a special case of the problem of type reconstruction for partially typed terms. Our result in this paper means that the restricted problem of type reconstruction for partially typed terms is still undecidable. Moreover, observation of the undecidability proof reveals that the typability problem is undecidable even for a predicative fragment of domain-free $\lambda 2$, called the rank 2 fragment 2017. This analysis also implies the involved result that the partial type reconstruction problem is still undecidable for the rank 2 fragment of second-order $\lambda$-calculus, contrary to the decidable typability for the rank 2 fragment of $\lambda 2$ in Curry style [17]. From the viewpoint of partially typed terms, we introduce fine structures between Church-style and Curry-style, including the domain-free style. In the uniform framework, we study the static properties of the $\lambda$-terms between the two styles. It is found that making polymorphic domains free and the use of type-holes [ ] are independently responsible for the undecidability of the partial type reconstruction problem. In this sense, this work can give a guide to the construction of typed languages with decidable type checking and typability.

\section{Curry-Style, Church-Style, and Domain-Free}

In Curry-style, terms are essentially type free 7816 , and types can be assigned by rules of a type theory if well-formed. Terms in the Church-style typed $\lambda$-calculus, on one hand, are originally defined only from variables uniquely type annotated [6]. Following Curry's philosophy, today one has the notion of pseudoterms [1] separated from a type theory. On the other hand, terms in domain-free (DF) style have domain-free $\lambda$-abstraction [4, and second-order $\lambda$-calculus in domain-free style can be regarded, in a sense, as an intermediate representation between à la Curry and à la Church, as shown in the following table:

Types $\sigma::=t|\sigma \rightarrow \sigma| \forall t . \sigma$

\section{Styles of (typed) $\lambda 2$-terms}

\begin{tabular}{|c||c|c|c|c|}
\hline \multicolumn{1}{|c||}{-(pseudo)terms } & object-var abst. & term app. & type-var abst. & type app. \\
\hline \hline Church-style & $\lambda x: \sigma . M$ & $M M$ & $\lambda t . M$ & $M[\sigma]$ \\
\hline Domain-Free & $\lambda x . N$ & $N N$ & $\lambda t . N$ & $N[\sigma]$ \\
\hline Curry-style & $\lambda x . U$ & $U U$ & & \\
\hline
\end{tabular}


We give a definition of domain-free $\lambda 2$-calculus. In terms of domain-free pure type systems [34, this domain-free system is constructed from sorted variables; a metavariable for variables of the first sort (term variables) is $x$ and a metavariable for variables of the second sort (type variables) is $t$. Then, on the basis of the sorted variables, type abstraction can be represented by $\lambda t$ rather than the traditional $\Lambda t$, and we also have explicit distinction between terms and types.

Type Assignment Rules for Domain-Free $\lambda 2$

$$
\begin{array}{cc} 
& \Gamma \vdash x: \Gamma(x) \\
\frac{\Gamma \vdash N_{1}: \sigma_{1} \rightarrow \sigma_{2} \quad \Gamma \vdash N_{2}: \sigma_{1}}{\Gamma \vdash N_{1} N_{2}: \sigma_{2}}(\rightarrow E) & \frac{\Gamma, x: \sigma_{1} \vdash N: \sigma_{2}}{\Gamma \vdash \lambda x \cdot N: \sigma_{1} \rightarrow \sigma_{2}}(\rightarrow I) \\
\frac{\Gamma \vdash N: \forall t . \sigma_{1}}{\Gamma \vdash N\left[\sigma_{2}\right]: \sigma_{1}\left[t:=\sigma_{2}\right]}(\forall E) & \frac{\Gamma \vdash N: \sigma}{\Gamma \vdash \lambda t . N: \forall t . \sigma}(\forall I)^{*}
\end{array}
$$

where $(\forall I)^{*}$ denotes the eigenvariable condition.

The introduction rules, $(\rightarrow I)$ and $(\forall I)$ can be coded, respectively, as domainfree $\lambda$-abstractions based on the distinction between the sorted variables. The elimination rules, $(\rightarrow E)$ and $(\forall E)$ can also be represented, respectively, by the pairs of two expressions, based on sorted variables. Hence, when well-typed terms of domain-free $\lambda 2$ are given, the type assignment rules are uniquely determined by the shape of the terms. From this syntactical property of terms, we have the natural generation lemma for domain-free $\lambda 2$.

Lemma 1 (Generation lemma) (1) If $\Gamma \vdash x: \sigma$, then $\Gamma(x)=\sigma$.

(2) If $\Gamma \vdash N_{1} N_{2}: \sigma$, then $\Gamma \vdash N_{1}: \sigma_{1} \rightarrow \sigma$ and $\Gamma \vdash N_{2}: \sigma_{1}$ for some $\sigma_{1}$.

(3) If $\Gamma \vdash \lambda x . N: \sigma$, then $\Gamma, x: \sigma_{1} \vdash N: \sigma_{2}$ and $\sigma \equiv \sigma_{1} \rightarrow \sigma_{2}$ for some $\sigma_{1}$ and $\sigma_{2}$.

(4) If $\Gamma \vdash \lambda t . N: \sigma$, then $\Gamma \vdash N: \sigma_{1}$ and $\sigma \equiv \forall t . \sigma_{1}$ together with $t \notin F V(\Gamma)$ for some $\sigma_{1}$.

(5) If $\Gamma \vdash N\left[\sigma_{1}\right]: \sigma$, then $\Gamma \vdash N: \forall t . \sigma_{2}$ and $\sigma \equiv \sigma_{2}\left[t:=\sigma_{1}\right]$ for some $\sigma_{2}$.

\section{Type Checking, Typability, and Type Inference for Domain-Free $\lambda 2$}

The problem of type inference is a problem; given a term $M$, are there a context $\Gamma$ and a type $\sigma$ such that $\Gamma \vdash M: \sigma$ is derivable? On one hand, the problem of strong type inference [30] is a problem; given a term $M$ and a context $\Gamma_{0}$, are there a context $\Gamma \supseteq \Gamma_{0}$ and a type $\sigma$ such that $\Gamma \vdash M: \sigma$ is derivable? The typability problem is a problem; given a term $M$ and a context $\Gamma$, is there a type $\sigma$ such that $\Gamma \vdash M: \sigma$ is derivable? Finally, the type checking problem is a problem; given a term $M$, a type $\sigma$, and a context $\Gamma$, is the judgement $\Gamma \vdash M: \sigma$ derivable? The problems of (strong) type inference, typability, and type checking are denoted, respectively, by ? $\vdash M: ?, \Gamma \vdash M: ?$, and $\Gamma \vdash M: \sigma$ ?. 
The problems of type checking, typability, and type inference for Curry and Church $\lambda 2$ are investigated by Jutting [31, Wells [32], and Schubert 2829], as shown in the following table:

Decidability for type checking, typability, and type inference of $\lambda 2$

\begin{tabular}{|c||c|c|c|}
\hline$\lambda 2$ & $\Gamma \vdash M: \sigma ?$ & $\Gamma \vdash M: ?$ & $?-M: ?$ \\
\hline \hline Church-style & yes [31] & yes $[31]$ & no [28] \\
\hline Domain-Free & $?_{1}$ & $?_{2}$ & $?_{3}$ \\
\hline Curry-style & no $[32]$ & no $[32]$ & no $[32]$ \\
\hline
\end{tabular}

In this paper, we will prove that in the table above, all of $?_{1}, ?_{2}$, and $?_{3}$ (case of strong type inference) are "no", i.e., undecidable1.

By the use of a type forgetful map, the three styles of judgements are equivalent in the following sense, where || is a domain erasing map $(|\lambda x: \sigma \cdot M|=$ $\lambda x .|M|)$, and \|\| is a type erasing map $(\|M \sigma\|=\|M\|,\|\lambda t . M\|=\|M\|)$ :

(1) If $\Gamma \vdash M: \sigma$ in Church style, then $\Gamma \vdash|M|: \sigma$ in domain-free style.

(2) If $\Gamma \vdash M: \sigma$ in domain-free style, then $\Gamma \vdash\|M\|: \sigma$ in Curry style.

The inverse directions say that there exists a term whose erasure is the same as the original term [15.

(-1) If $\Gamma \vdash M: \sigma$ in domain-free style, then there exists $M_{1}$ such that $\Gamma \vdash M_{1}: \sigma$ in Church style and $\left|M_{1}\right| \equiv M$.

(-2) If $\Gamma \vdash M: \sigma$ in Curry style, then there exists $M_{2}$ such that $\Gamma \vdash M_{2}: \sigma$ in domain-free style and $\left\|M_{2}\right\| \equiv M$.

For the problems above, however, the inverse directions are not straightforward, since the forgetful maps are not one-to-one. We have to check that the given term is the same as an erasure of some term, or that the given term has the same type as that of the erasure, see also Section 7 . Here, we will directly study the problems for domain-free $\lambda 2$.

On the basis of the generation lemma (Lemma 1), we first observe that the (strong) type inference problem for domain-free $\lambda 2$ is reduced to the typability problem, and the typability problem for domain-free $\lambda 2$ is reduced to the type checking problem.

Lemma $2 \exists \Gamma . \exists \sigma . \Gamma, \Gamma_{0} \vdash M: \sigma$ in DF $\lambda 2 \Longleftrightarrow \exists \sigma . \Gamma_{0} \vdash \lambda \vec{x} . M: \sigma$ in DF $\lambda 2$ $\Longleftrightarrow \quad \Gamma_{0} \vdash(\lambda x . \lambda y . y)(\lambda \vec{x} . M): t \rightarrow t$ in $\mathrm{DF} \lambda 2$

\section{Terms with Partial Type Annotations}

In order to study static properties of $\lambda$-terms in a uniform framework, we introduce partially typed terms (preterms) and type assignment rules for preterms.

Partially typed terms (preterms), denoted by $P, Q$, are: $P::=x|\lambda x: \sigma . P| P P|\lambda t . P| P[\sigma]^{a}|\lambda x . P| P[]^{a}$

1 After completing [11]12, a correspondence with Gilles Barthe informed the author that all of ? are independently proved undecidable in [5. For the detail, see also the footnote in Section 7. 
where the mark []$^{a}$ must be left to indicate that a type has been erased. Moreover, the label $a$ in [ $]^{a}$ will be used to identify the occurrences of [ ], i.e., the type-holes [ ] with the same label should be obtained by erasing the same type. This annotation is a natural constraint to the traditional definition of preterms [225] and gives a useful information. The use of our type-holes [ $]^{a}$ plays the same role as existential quantification over types, see also Proposition 4. We often omit the annotation of a type-hole unless it is necessary to identify the occurrences.

We say that a preterm $P$ is a partial erasure of $M$ in Church-style, denoted by $P \preceq_{1} M ; \Delta$ if it is derived by the following rules, where $\Delta$ is a mapping from an annotation to a type:

$$
\begin{array}{cc}
\overline{x \preceq_{1} x ;} \text { (var) } \\
\frac{P \preceq_{1} M ; \Delta}{\lambda x: \sigma . P \preceq_{1} \lambda x: \sigma . M ; \Delta} \text { (abst-var) } & \frac{P \preceq_{1} M ; \Delta}{\lambda x . P \preceq_{1} \lambda x: \sigma \cdot M ; \Delta} \text { (abst-df) } \\
\frac{P_{1} \preceq_{1} M_{1} ; \Delta \quad P_{2} \preceq_{1} M_{2} ; \Delta}{P_{1} P_{2} \preceq_{1} M_{1} M_{2} ; \Delta} \text { (app) } & \frac{P \preceq_{1} M ; \Delta}{\lambda t . P \preceq_{1} \lambda t . M ; \Delta} \text { (abst-type) } \\
\left.\frac{P \preceq_{1} M ; \Delta}{P[\sigma]^{a} \preceq_{1} M[\sigma]^{a} ; \Delta, \sigma^{a}} \text { (app-type }\right)^{\sharp} & \left.\frac{P \preceq_{1} M ; \Delta}{P[]^{a} \preceq_{1} M[\sigma]^{a} ; \Delta, \sigma^{a}} \text { (app-hole) }\right)^{\sharp}
\end{array}
$$

Here, $\sharp$ denotes the condition such that if $\Delta(a) \neq \emptyset$ then $\Delta(a)=\sigma$.

We now consider special cases of preterms, which give fine structures between Church-style and Curry-style, as follows:

(1) Domain-free terms:

Preterms which are derived by the use of all the rules but (abst-var) and (apphole).

(2) [ ]-application terms:

Preterms which are derived by all the rules but (abst-df) and (app-type).

(3) DF\&[ ] terms:

Preterms which are derived by all the rules but (abst-var) and (app-type).

A term in Curry-style is here regarded as a full erasure that is obtained from a term of DF\&[ ] by deleting both $\lambda t$ and [ ].

We also say that a preterm $P_{1}$ is a partial erasure of a preterm $P_{2}$, denoted by $P_{1} \preceq P_{2}$ if it is derived by the following rules:

$$
\begin{aligned}
& x \preceq x \quad \frac{P_{1} \preceq P_{2}}{\lambda x: \sigma \cdot P_{1} \preceq \lambda x: \sigma \cdot P_{2}} \\
& \frac{P_{1} \preceq P_{2}}{\lambda x \cdot P_{1} \preceq \lambda x: \sigma \cdot P_{2}} \quad \frac{P_{1} \preceq P_{2}}{\lambda x \cdot P_{1} \preceq \lambda x \cdot P_{2}} \quad \frac{P_{1} \preceq Q_{1} \quad P_{2} \preceq Q_{2}}{P_{1} P_{2} \preceq Q_{1} Q_{2}} \\
& \frac{P_{1} \preceq P_{2}}{\lambda t . P_{1} \preceq \lambda t . P_{2}} \quad \frac{P_{1} \preceq P_{2}}{P_{1}[\sigma] \preceq P_{2}[\sigma]} \quad \frac{P_{1} \preceq P_{2}}{P_{1}[] \preceq P_{2}[\sigma]} \quad \frac{P_{1} \preceq P_{2}}{P_{1}[] \preceq P_{2}[]}
\end{aligned}
$$

It is clear that $\preceq$ constitutes a partial order on preterms. 
We next define type assignment rules for preterms:

$$
\begin{aligned}
& \Gamma \vdash_{p} x: \Gamma(x) ; \Delta \\
& \frac{\Gamma \vdash_{p} P_{1}: \sigma_{1} \rightarrow \sigma_{2} ; \Delta \quad \Gamma \vdash_{p} P_{2}: \sigma_{1} ; \Delta}{\Gamma \vdash_{p} P_{1} P_{2}: \sigma_{2} ; \Delta}(\rightarrow E) \\
& \frac{\Gamma, x: \sigma_{1} \vdash_{p} P: \sigma_{2} ; \Delta}{\Gamma \vdash_{p} \lambda x: \sigma_{1} \cdot P: \sigma_{1} \rightarrow \sigma_{2} ; \Delta}\left(\rightarrow I_{1}\right) \quad \frac{\Gamma, x: \sigma_{1} \vdash_{p} P: \sigma_{2} ; \Delta}{\Gamma \vdash_{p} \lambda x \cdot P: \sigma_{1} \rightarrow \sigma_{2} ; \Delta}\left(\rightarrow I_{2}\right) \\
& \frac{\Gamma \vdash_{p} P: \forall t . \sigma_{1} ; \Delta}{\Gamma \vdash_{p} P\left[\sigma_{2}\right]^{a}: \sigma_{1}\left[t:=\sigma_{2}\right] ; \Delta}\left(\forall E_{1}\right)^{\sharp \sharp} \quad \frac{\Gamma \vdash_{p} P: \forall t . \sigma_{1} ; \Delta}{\Gamma \vdash_{p} P[]^{a}: \sigma_{1}\left[t:=\sigma_{2}\right] ; \Delta}\left(\forall E_{2}\right)^{\sharp \sharp} \\
& \frac{\Gamma \vdash_{p} P: \sigma ; \Delta}{\Gamma \vdash_{p} \lambda t . P: \forall t . \sigma ; \Delta}(\forall I)^{*}
\end{aligned}
$$

where $(\forall I)^{*}$ denotes the eigenvariable condition, and $\sharp \sharp$ does $\Delta(a)=\sigma_{2}$.

We may write $\Gamma \vdash_{p} P: \sigma$ for $\Gamma \vdash_{p} P: \sigma ; \Delta$ and [ ] for [ ] ${ }^{a}$, unless it is necessary to use annotations for type-holes. Since a well-typed preterm can be uniquely decomposed by one of the assignment rules, we have the generation lemma below:

Lemma 3 (Generation lemma) (1) If $\Gamma \vdash_{p} x: \sigma ; \Delta$, then $\Gamma(x)=\sigma$.

(2) If $\Gamma \vdash_{p} P_{1} P_{2}: \sigma ; \Delta$, then $\Gamma \vdash_{p} P_{1}: \sigma_{1} \rightarrow \sigma ; \Delta$ and $\Gamma \vdash P_{2}: \sigma_{1} ; \Delta$ for some $\sigma_{1}$.

(3) If $\Gamma \vdash_{p} \lambda x: \sigma_{1} . P: \sigma ; \Delta$, then $\Gamma, x: \sigma_{1} \vdash_{p} P: \sigma_{2} ; \Delta$ and $\sigma \equiv \sigma_{1} \rightarrow \sigma_{2}$ for some $\sigma_{2}$.

(4) If $\Gamma \vdash_{p} \lambda x . P: \sigma ; \Delta$, then $\Gamma, x: \sigma_{1} \vdash_{p} P: \sigma_{2} ; \Delta$ and $\sigma \equiv \sigma_{1} \rightarrow \sigma_{2}$ for some $\sigma_{1}$ and $\sigma_{2}$.

(5) If $\Gamma \vdash_{p} \lambda$ t.P : $\sigma ; \Delta$, then $\Gamma \vdash_{p} P: \sigma_{1} ; \Delta$ together with $\sigma \equiv \forall t . \sigma_{1}$ and $t \notin F V(\Gamma)$ for some $\sigma_{1}$.

(6) If $\Gamma \vdash_{p} P\left[\sigma_{1}\right]^{a}: \sigma ; \Delta$, then $\Gamma \vdash P: \forall t . \sigma_{2} ; \Delta$ together with $\sigma \equiv \sigma_{2}\left[t:=\sigma_{1}\right]$ and $\Delta(a)=\sigma_{1}$ for some $\sigma_{2}$.

(7) If $\Gamma \vdash_{p} P[]^{a}: \sigma ; \Delta$, then $\Gamma \vdash P: \forall t . \sigma_{2} ; \Delta$ together with $\sigma \equiv \sigma_{2}\left[t:=\sigma_{1}\right]$ and $\Delta(a)=\sigma_{1}$ for some $\sigma_{2}$.

The above mentioned generation lemma allows to deduce the following relationship between Church-style judgements and judgements of preterms.

Lemma 4 (1) If we have $\Gamma \vdash_{p} P_{1}: \sigma$, then $\Gamma \vdash_{p} P_{2}: \sigma$ for any $P_{2} \preceq P_{1}$.

(2) If we have $\Gamma \vdash_{p} P: \sigma ; \Delta$, then $\Gamma \vdash M: \sigma$ in Church style for some $M \succeq_{1} P ; \Delta$.

The problem of partial type reconstruction [25 26] is a problem; given a context $\Gamma$ and a preterm $P$, is there a Church-style term $M$ such that $\Gamma \vdash M: \sigma$ holds in Church-style for some type $\sigma$ and that $P \preceq_{1} M ; \Delta$ for some $\Delta$ ? From the lemma 4, the partial type reconstruction problem is equivalent to the following typability problem:

Given a context $\Gamma$ and a preterm $P$, is there a type $\sigma$ such that $\Gamma \vdash_{p} P: \sigma ; \Delta$ is derivable for some $\Delta$ ?

We say that a preterm $P$ is a normal form if $P$ contains a subterm in the form of neither $\left(\lambda x: \sigma . P_{1}\right) P_{2},\left(\lambda x . P_{1}\right) P_{2},\left(\lambda t . P_{1}\right) \sigma$, nor $\left(\lambda t . P_{1}\right)[]$. 


\section{Type Inference is Undecidable for Domain-Free $\lambda 2$}

In this section, we prove that the problem of strong type inference for domainfree $\lambda 2$ is undecidable. To show this, we demonstrate a stronger result such that the problem of strong type inference is undecidable for the predicative fragment of domain-free $\lambda 2$, called domain-free ML. Strictly speaking, the following system is a subsystem of the so-called ML, however such a subsystem is enough to establish the undecidability.

Domain-free (DF) ML:

Monotypes $\tau::=t \mid \tau \rightarrow \tau \quad$ Polytypes $\sigma::=\tau \mid \forall t . \sigma$

Contexts $\Gamma::=\langle\rangle \mid x: \sigma, \Gamma$

Terms $M::=x|\lambda x . M| M M \mid x\left[\tau_{1}\right] \cdots\left[\tau_{n}\right]$

Type Assignment Rules

$$
\begin{gathered}
\Gamma(x)=\forall t_{1} \cdots t_{n} . \tau \\
\frac{\Gamma \vdash x\left[\tau_{1}\right] \cdots\left[\tau_{n}\right]: \tau\left[t_{1}:=\tau_{1}, \cdots t_{n}:=\tau_{n}\right]}{\Gamma}(n \geq 0) \\
\frac{\Gamma, x: \tau_{1} \vdash M: \tau_{2}}{\Gamma \vdash \lambda x \cdot M: \tau_{1} \rightarrow \tau_{2}} \quad \frac{\Gamma \vdash M_{1}: \tau_{1} \rightarrow \tau_{2}}{\Gamma \vdash M_{1} M_{2}: \tau_{2}} \quad \Gamma \vdash M_{2}: \tau_{1}
\end{gathered}
$$

Remarked that [ ]-application ML can also be defined similarly.

We first introduce a restricted second-order unification problem for wellformed second-order expressions, which are defined from monotypes $\tau$, binary function constant $\rightarrow$, and $n$-arity second-order function variable $X(n \geq 0)$ whose arguments contain no variables. Such terms for the second-order unification are denoted by $T$ or $U$. A well-formed expression is defined as follows:

(1) A type variable $t$ is a well-formed expression.

(2) If $X$ is an $n$-arity variable $(n \geq 0)$ and $\tau_{i}(1 \leq i \leq n)$ are monotypes, then $X \tau_{1} \cdots \tau_{n}$ is well-formed.

(3) If $T_{1}$ and $T_{2}$ are well-formed, then so is $T_{1} \rightarrow T_{2}$.

Given a well-formed expression $T$, a set of (unification) variables in $T$ denoted by $u \operatorname{Var}(T)$ and a set of constants in $T$ denoted by $\operatorname{Con}(T)$ are defined, respectively, as follows:

$u \operatorname{Var}(t)=\emptyset ; \quad u \operatorname{Var}\left(X \tau_{1} \cdots \tau_{n}\right)=\{X\}(n \geq 0) ;$

$u \operatorname{Var}\left(T_{1} \rightarrow T_{2}\right)=u \operatorname{Var}\left(T_{1}\right) \cup u \operatorname{Var}\left(T_{2}\right)$.

$\operatorname{Con}(t)=\{t\} ; \quad \operatorname{Con}\left(X \tau_{1} \cdots \tau_{n}\right)=\emptyset(n \geq 0) ;$

$\operatorname{Con}\left(T_{1} \rightarrow T_{2}\right)=\operatorname{Con}\left(T_{1}\right) \cup \operatorname{Con}\left(T_{2}\right)$.

Given well-formed $T_{1}$ and $T_{2}$. Let $u \operatorname{Var}\left(T_{1}, T_{2}\right)$ be $u \operatorname{Var}\left(T_{1}\right) \cup u \operatorname{Var}\left(T_{2}\right)=$ $\left\{X_{1}, \cdots, X_{n}\right\}$. The unification problem $\left(T_{1} \doteq T_{2}\right)$ is a problem to find wellformed $U_{i}$ for each $X_{i}(1 \leq i \leq n)$, such that

(1) Let $X_{i}$ be $k(i)$-arity variable, and $S$ be a substitution such that $\left[X_{1}:=\lambda t_{1} \cdots t_{k(1)} \cdot U_{1}, \cdots, X_{n}:=\lambda t_{1} \cdots t_{k(n)} \cdot U_{n}\right]$. Then $S\left(T_{1}\right)={ }_{\beta} S\left(T_{2}\right)$ holds.

(2) We have $u \operatorname{Var}\left(U_{i}\right)=\emptyset$ for $1 \leq i \leq n$.

If we have a substitution $S$ such that the above (1) and (2) are satisfied, then we say that $T_{1}$ and $T_{2}$ are unifiable, and that the unification problem has an 
answer $S$. In this case, from the definition, there exists a monotype $\tau$ such that $S\left(T_{1}\right)={ }_{\beta} \tau={ }_{\beta} S\left(T_{2}\right)$.

Theorem 1 (Schubert 28]). The second-order unification problem on the wellformed expressions is undecidable.

Schubert [28] has proved that the halting problem for two-counter automata is reduced to the unification problem, where a two-counter automaton can simulate an arbitrary Turing machine.

In order to give a reduction from the unification problem to the problem of type inference for domain-free ML, we first define a (pre)context $\Sigma$. The context itself may not be an ML-context, but it becomes an ML-context under some substitution if unifiable. This can be justified, since the reduction is formalized as follows: the unification problem $T_{1} \doteq T_{2}$ has an answer if and only if there exist $\Gamma$ and $\tau$ such that $\Gamma, \Gamma_{0} \vdash M: \tau$ in domain-free ML. Here, $\Gamma_{0}$ and $M$ are given by $T_{1}$ and $T_{2}$, where $\Gamma_{0}$ consists only of monotypes in $\operatorname{Con}\left(T_{1}, T_{2}\right)$. Only if $T_{1}$ and $T_{2}$ are unifiable, say the unifier $S$, then the ML-context $\Gamma$ can be obtained as a subcontext of $S(\Sigma)$, such that $S(\Sigma)=\Gamma, \Gamma_{0}$. Moreover, the monotype $\tau$ can also be obtained as a substitution instance (of $t y(\cdot)$ defined below) by $S$.

Given a well-formed $T$, then we construct the context $\Sigma[T]$, such that

(1) For each $t \in \operatorname{Con}(T), t$ is inhabited in $\Sigma[T]$, i.e., $\Sigma[T](x)=t$ for some $x$.

(2) For each $n$-arity variable $X \in u \operatorname{Var}(T)$ where $n \geq 0$, the universal closure $\forall t_{1} \cdots t_{n} \cdot\left(X t_{1} \cdots t_{n}\right)$ is inhabited in $\Sigma[T]$.

$\Sigma\left[T_{1}, T_{2}\right]$ is also defined similarly, and we simply write $\Sigma$ for $\Sigma\left[T_{1}, T_{2}\right]$.

Let $T_{1}$ and $T_{2}$ be well-formed. Given a second-order unification problem $T_{1} \doteq T_{2}$, then, following Pfenning [26], we construct a term of domain-free ML by the use of the following $\mathcal{U} \mathcal{T}$ and $\mathcal{T} \mathcal{I}$ :

$\mathcal{U T}\left(\Sigma\left[T_{1}, T_{2}\right] ; T_{1} \doteq T_{2}\right)=$

$$
\lambda z_{1} \cdot \lambda z_{2} . \lambda f . f z_{1}\left(f z_{2}\left(\lambda g . g\left(\mathcal{T I}\left(\Sigma ; z_{1} ; T_{1}\right)\right)\left(\mathcal{T} \mathcal{I}\left(\Sigma ; z_{2} ; T_{2}\right)\right)\right)\right)
$$

where $\mathcal{T} \mathcal{I}(\Sigma ; z ; T)$ is defined by induction on $T$ :

(1) $\mathcal{T} \mathcal{I}(\Sigma ; z ; t)=\lambda f . f z(f x(\lambda g . g))$ where $\Sigma(x)=t \in \operatorname{Con}\left(T_{1}, T_{2}\right)$

(2) $\mathcal{T} \mathcal{I}\left(\Sigma ; z ; X \tau_{1} \cdots \tau_{n}\right)=\lambda f . f z\left(f\left(x\left[\tau_{1}\right] \cdots\left[\tau_{n}\right]\right)(\lambda g . g)\right)$

where $\Sigma(x)=\forall t_{1} \cdots t_{n} \cdot\left(X t_{1} \cdots t_{n}\right)$

(3) $\mathcal{T} \mathcal{I}\left(\Sigma ; z ; T_{1} \rightarrow T_{2}\right)=$

$$
\lambda z_{1} \cdot \lambda z_{2} \cdot \lambda f . f\left(z z_{1}\right)\left(f z_{2}\left(\lambda g . g\left(\mathcal{T I}\left(\Sigma ; z_{1} ; T_{1}\right)\right)\left(\mathcal{T} \mathcal{I}\left(\Sigma ; z_{2} ; T_{2}\right)\right)\right)\right)
$$

Remarks 1 The reduction via $\mathcal{U} \mathcal{T}$ and $\mathcal{T} \mathcal{I}$ gives a $\beta$-normal term.

The translation $\mathcal{T} \mathcal{I}(\Sigma ; z ; T)$ says that type of $z$ would be a substitution instance of $T$, see Lemma 5 below.

We next construct $\operatorname{ty}(T)$ that is a type of $\mathcal{T} \mathcal{I}(\Sigma ; z ; T)$. Although $\operatorname{ty}(T)$ itself may not be a monotype, it becomes a monotype under some substitution if unifiable. Here of course we assume that we have countably many type variables to use a fresh type variable $t$ for each application of the following definition:

(0) $\operatorname{ty}(\tau)=(\tau \rightarrow(t \rightarrow t) \rightarrow t \rightarrow t) \rightarrow t \rightarrow t$ for $\tau \in \operatorname{Con}(T)$;

(1) $\operatorname{ty}\left(X \tau_{1} \cdots \tau_{n}\right)=\left(\left(X \tau_{1} \cdots \tau_{n}\right) \rightarrow(t \rightarrow t) \rightarrow t \rightarrow t\right) \rightarrow t \rightarrow t$ where $n \geq 0$;

(2) $\operatorname{ty}\left(T_{1} \rightarrow T_{2}\right)=T_{1} \rightarrow T_{2} \rightarrow\left(T_{2} \rightarrow A \rightarrow A\right) \rightarrow A$ where $A \equiv\left(t y\left(T_{1}\right) \rightarrow t y\left(T_{2}\right) \rightarrow t\right) \rightarrow t$. 
Lemma $5 S(\Sigma[T]), x: \tau \vdash \mathcal{T} \mathcal{I}(\Sigma[T] ; x ; T): S(t y(T))$ in domain-free ML if and only if $S(T)={ }_{\beta} \tau$.

Proof. By induction on $T$. We show the following two cases:

(1) Case of $T \equiv X \tau_{1} \cdots \tau_{n}$

Let $S(\Sigma(y))$ be $\forall t_{1} \cdots t_{n} \cdot\left((S X) t_{1} \cdots t_{n}\right)$. We have

$S(\Sigma), x: \tau \vdash \lambda f . f x\left(f\left(y\left[\tau_{1}\right] \cdots\left[\tau_{n}\right]\right)(\lambda g . g)\right):$

$$
\left(\left((S X) \tau_{1} \cdots \tau_{n}\right) \rightarrow(t \rightarrow t) \rightarrow t \rightarrow t\right) \rightarrow t \rightarrow t
$$

in domain-free ML. Here, types of $x$ and $y\left[\tau_{1}\right] \cdots\left[\tau_{n}\right]$ must be equal. That is, $\tau={ }_{\beta}\left((S X) \tau_{1} \cdots \tau_{n}\right)$.

(2) Case of $T \equiv T_{1} \rightarrow T_{2}$

From the definition, in domain-free ML we have

$S(\Sigma), x: \tau \vdash \lambda z_{1} . \lambda z_{2} . \lambda f . f\left(x z_{1}\right)\left(f z_{2}\left(\lambda g . g\left(\mathcal{T I}\left(\Sigma ; z_{1} ; T_{1}\right)\right)\left(\mathcal{T I}\left(\Sigma ; z_{2} ; T_{2}\right)\right)\right)\right):$

$$
S\left(T_{1}\right) \rightarrow S\left(T_{2}\right) \rightarrow\left(S\left(T_{2}\right) \rightarrow A \rightarrow A\right) \rightarrow A
$$

where $A \equiv\left(S\left(t y\left(T_{1}\right)\right) \rightarrow S\left(t y\left(T_{2}\right)\right) \rightarrow t\right) \rightarrow t$.

Then, we also have

$S(\Sigma), x: \tau, z_{1}: S\left(T_{1}\right), z_{2}: S\left(T_{2}\right) \vdash$

$\lambda f . f\left(x z_{1}\right)\left(f z_{2}\left(\lambda g . g\left(\mathcal{T I}\left(\Sigma ; z_{1} ; T_{1}\right)\right)\left(\mathcal{T} \mathcal{I}\left(\Sigma ; z_{2} ; T_{2}\right)\right)\right)\right):\left(S\left(T_{2}\right) \rightarrow A \rightarrow A\right) \rightarrow A$.

Here, from the induction hypotheses, we have the following:

$S(\Sigma), z_{1}: \tau_{3} \vdash \mathcal{T I}\left(\Sigma ; z_{1} ; T_{1}\right): S\left(t y\left(T_{1}\right)\right) \quad$ iff $\quad \tau_{3}={ }_{\beta} S\left(T_{1}\right)$

$S(\Sigma), z_{2}: \tau_{4} \vdash \mathcal{T} \mathcal{I}\left(\Sigma ; z_{2} ; T_{2}\right): S\left(t y\left(T_{2}\right)\right) \quad$ iff $\quad \tau_{4}=_{\beta} S\left(T_{2}\right)$

Now, type of $\left(x z_{1}\right)$ and $z_{2}$ must be equal, i.e., $\tau={ }_{\beta} S\left(T_{1}\right) \rightarrow S\left(T_{2}\right)$.

Lemma 6 (main lemma) $S\left(T_{1}\right)={ }_{\beta} S\left(T_{2}\right)$ if and only if $S(\Sigma) \vdash \mathcal{U T}\left(\Sigma ; T_{1} \doteq T_{2}\right): S\left(t y\left(T_{1} \rightarrow T_{2}\right)\right)$ in domain-free ML.

Proof. $S(\Sigma) \vdash \mathcal{U T}\left(\Sigma ; T_{1} \doteq T_{2}\right): S\left(t y\left(T_{1} \rightarrow T_{2}\right)\right)$

iff $S(\Sigma), z_{1}: S\left(T_{1}\right), z_{2}: S\left(T_{2}\right) \vdash \lambda f . f z_{1}\left(f z_{2}\left(\lambda g . g\left(\mathcal{T} \mathcal{I}\left(\Sigma ; z_{1} ; T_{1}\right)\right)\left(\mathcal{T} \mathcal{I}\left(\Sigma ; z_{2} ; T_{2}\right)\right)\right)\right)$

$:\left(S\left(T_{2}\right) \rightarrow A \rightarrow A\right) \rightarrow A$ where $A \equiv\left(S\left(t y\left(T_{1}\right)\right) \rightarrow S\left(t y\left(T_{2}\right)\right) \rightarrow t\right) \rightarrow t$

iff (Lemma [5) $S\left(T_{1}\right)={ }_{\beta} S\left(T_{2}\right)$.

Proposition 1 The unification problem on the well-formed expressions is reduced to the problem of strong type inference for domain-free ML. In other words, $S\left(T_{1}\right)={ }_{\beta} S\left(T_{2}\right) \Longleftrightarrow \exists \Gamma . \exists \tau . \Gamma, \Gamma_{0}^{T_{1,2}} \vdash M^{T_{1,2}}: \tau$ in domain-free ML.

Proof. $(\Rightarrow)$ : From Lemma 6, $\Gamma_{0}^{T_{1,2}}$ and $M^{T_{1,2}}$ are determined by $T_{1}$ and $T_{2}$, such that for each $t \in \operatorname{Con}\left(T_{1}, T_{2}\right)$, we have $\Gamma_{0}(x)=t$ for some $x$, and that $M=\mathcal{U T}\left(\Sigma ; T_{1} \doteq T_{2}\right)$. Then the unifier $S$ gives $\Gamma$ and $\tau$, respectively, such that $S\left(\Sigma\left[T_{1}, T_{2}\right]\right)=\Gamma, \Gamma_{0}$ and $S\left(t y\left(T_{1} \rightarrow T_{2}\right)\right)=\tau$.

$(\Leftarrow)$ : Given $\Gamma_{0}^{T_{1,2}}$ and $M^{T_{1,2}}$, and assume that there exist $\tau$ and $\Gamma$ such that $\Gamma=\left\{x_{1}: \tau_{1}, \cdots, x_{m}: \forall t_{1} \cdots t_{n} \cdot \tau_{m}\right\}$. For each $X_{i} \in u \operatorname{Var}\left(T_{1}, T_{2}\right)$, assume that $\Sigma\left(x_{1}\right)=X_{1}, \cdots, \Sigma\left(x_{m}\right)=\forall t_{1} \cdots t_{n} \cdot\left(X_{m} t_{1} \cdots t_{n}\right)$. Then an answer to the trivial second-order matching problem such that $X_{1} \doteq \tau_{1}, \cdots,\left(X_{m} t_{1} \cdots t_{n}\right) \doteq \tau_{m}$ finds a matching $S$ for $t y\left(T_{1} \rightarrow T_{2}\right) \doteq \tau$, since if $S(\Sigma[T]), x: \tau_{0} \vdash \mathcal{T} \mathcal{I}(\Sigma[T] ; x ; T): \tau$ for some $\tau$, then $S(\operatorname{ty}(T))={ }_{\beta} \tau$. From Lemma [6] the unifier $S$ is an answer to the unification problem $T_{1} \doteq T_{2}$. 
Proposition 2 (no $(\mathrm{nf}, \mathrm{str})^{P(2)}$ ) The problem of strong type inference is undecidable for domain-free $\mathrm{ML}$, even when the given term is a normal form.

Proof. From Theorem 11 Proposition 1, and Remark 1.

Remarks 2 (no(nf, str) ${ }^{h}$ ) The proof of undecidability for strong type inference is also applicable to that for DF\&[ ] terms in ML-fragment.

Theorem $2\left(\right.$ no $^{T}\left[\mathbf{2}, \operatorname{no}(\mathrm{nf})^{T[\mathbf{2}}\right)$. Type checking, typability, and strong type inference are undecidable for domain-free $\lambda 2$.

Proof. From Proposition 2 and Lemma 2 Moreover, even in the case where the given term is a normal form, typability and strong type inference for domain-free $\lambda 2$ are still undecidable.

The problem of typability becomes undecidable for some predicative extension of the domain-free ML. We introduce a predicative fragment of domain-free $\lambda 2$, called domain-free $\mathrm{ML}_{2}$. This extension allows us to abstract a term variable with a polymorphic type $\sigma$ (polymorphic abstraction), but not to apply a polymorphic function to a polymorphic type (i.e., only to a monomorphic type $\tau)$. For this purpose, an extension of type schemes is introduced as follows:

$$
\rho::=\tau \mid \sigma \rightarrow \rho
$$

This type $\rho$ belongs to the so-called S(2)-class in [17, which is a special form of restrict types of rank 2 [20]. Following [17], a set of types with rank- $k$, denoted by $R(k)$ is defined as follows:

$R(0)=$ set of monotypes;

$R(k+1)=R(k)|R(k) \rightarrow R(k+1)| \forall t . R(k+1)$.

Domain-free (DF) $\mathrm{ML}_{2}$-fragment:

$$
\begin{gathered}
\Gamma(x)=\forall t_{1} \cdots t_{n} \cdot \tau \\
\frac{\Gamma \vdash M_{1}: \tau_{1} \rightarrow \tau_{2} \quad \Gamma \vdash M_{2}: \tau_{1}}{\Gamma \vdash M_{1} M_{2}: \tau_{2}} \quad \frac{\Gamma, x: \sigma \vdash M: \rho}{\Gamma \vdash \lambda x \cdot M: \sigma \rightarrow \rho} \\
\frac{\left.1 \tau_{1}\right]: \tau\left[t_{1}:=\tau_{1}, \cdots t_{n}:=\tau_{n}\right]}{\Gamma \vdash \rho}
\end{gathered}
$$

The problem of strong type inference in domain-free ML can be reduced to the typability problem in domain-free $\mathrm{ML}_{2}$. That is, let $\left\{x_{1}, \cdots, x_{n}\right\}$ be a set of free variables in $M ; \exists \sigma_{1} \cdots \sigma_{n} \cdot \exists \tau . \Gamma_{0}, x_{1}: \sigma_{1}, \cdots, x_{n}: \sigma_{n} \vdash M: \tau$ in DF ML-fragment if and only if $\exists \sigma_{1} \cdots \sigma_{n} . \exists \tau . \Gamma_{0} \vdash \lambda x_{1} \cdots x_{n} . M: \sigma_{1} \rightarrow \cdots \rightarrow \sigma_{n} \rightarrow \tau$ in DF $\mathrm{ML}_{2}$-fragment. From Proposition[2] we can obtain that the typability problem is undecidable for the rank 2 fragment of domain-free $\lambda 2$. This result means that the partial type reconstruction problem is still undecidable even for the rank 2 fragment of $\lambda 2$.

Corollary 1 (no(nf) ${ }^{\text {1 }}$ ) The typability problem for $\mathrm{DF} \mathrm{ML}_{2}$ is undecidable. 


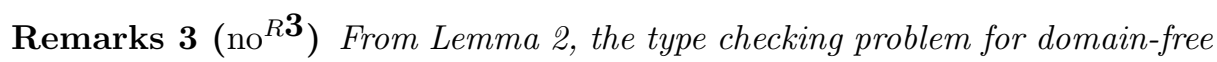
terms becomes undecidable at rank 3 .

Remarks 4 (no(nf) ${ }^{14}$ ) From remark [2, the typability problem for DF\&[] terms in $\mathrm{ML}_{2}$ is undecidable.

\section{Static Properties of [ ]-Application}

In this section, we study type inference, typability, and type checking with respect to [ ]-application terms. That is, the preterms have the following structure: $P::=x|\lambda x: \sigma . P| P P|\lambda t . P| P[]^{a}$

We first give a translation \lceil\rceil from a term in Church-style to [ ]-application terms, which is motivated by the translation [5] from Church-style to domainfree style. Let id $\equiv \lambda t . \lambda x: t . x$. Then id $: \forall t .(t \rightarrow t)$.

$\lceil x\rceil=x ; \quad\lceil\lambda x: \sigma \cdot M\rceil=\lambda x: \sigma .\lceil M\rceil ;$

$\left\lceil M_{1} M_{2}\right\rceil=\left\lceil M_{1}\right\rceil\left\lceil M_{2}\right\rceil ; \quad\lceil\lambda t . M\rceil=\lambda t .\lceil M\rceil ;$

$\lceil M[\sigma]\rceil=\left(\lambda v: \sigma \rightarrow \sigma .\lceil M\rceil[]^{a}\right)\left(\lambda x: \sigma . \mathrm{id}[]^{a} x\right)$ where $v$ is a fresh variable.

For any term $M$ in Church-style, $\lceil M\rceil$ is a preterm of [ ]-applications.

Proposition $3 \Gamma \vdash M: \sigma$ in Church-style $\lambda 2$ iff $\Gamma \vdash_{p}\lceil M\rceil: \sigma$.

The problem of type inference for $\lambda 2$ in Church-style is reduced to the (strong) type inference problem for preterms of [ ]-applications. Hence from the undecidability of type inference for $\lambda 2$ in Church-style [29], the (strong) type inference problem for preterms of [ ]-applications is undecidable.

Theorem 3 (no ${ }^{T[3}$ ). The (strong) type inference problem for [ ]-application terms is undecidable.

Remarks 5 (no ${ }^{\text {55] }}$ ) Following [28], since the type inference problem is undecidable for ML-fragment in Church-style, the (strong) type inference problem for [ ]-application terms becomes undecidable in the ML-fragment.

By the use of [ ]-applications, we give a translation which moves existentially quantified types in the context to the right-hand side of $\vdash_{p}$.

Proposition $4 \exists \sigma_{1} \cdots \sigma_{n} \cdot \exists \sigma . x_{1}: \sigma_{1}, \cdots, x_{n}: \sigma_{n} \vdash_{p} P: \sigma$ if and only if $\exists \sigma . y_{1}: \forall t . t, \cdots, y_{n}: \forall t . t \vdash_{p}\langle P\rangle: \sigma$,

where $\left\langle x_{i}\right\rangle=y_{i}[]^{a_{i}}, \quad\langle x\rangle=x$ if $x \not \equiv x_{i}(1 \leq i \leq n)$;

$\langle\lambda x: \sigma . P\rangle=\lambda x: \sigma .\langle P\rangle ; \quad\left\langle P_{1} P_{2}\right\rangle=\left\langle P_{1}\right\rangle\left\langle P_{2}\right\rangle$;

$\langle\lambda t . P\rangle=\lambda t .\langle P\rangle ; \quad\langle P[\sigma]\rangle=\langle P\rangle[\sigma] ; \quad\langle P[]\rangle=\langle P\rangle[]$.

The variable $x$ declared in $\Gamma$ is translated to $y[]^{a}$, and the new variable $y$ appears only in the form of $y[]^{a}$ in $\langle P\rangle$. The problem of type inference for [ ]application terms is reduced to the typability problem for [ ]-application terms. From Propositions [3 and 4, the typability problem for [ ]-application terms is essentially equivalent to type inference in Church-style. From Theorem [3, the typability problem for [ ]-application terms becomes undecidable. 
Theorem 4 (no $\left.{ }^{T 4}\right)$. The typability problem for []-application terms is undecidable.

Remarks 6 (no ${ }^{16}$ ) From Remark [5, the typability problem for [ ]-application terms becomes undecidable at rank 2. This rank 2 fragment is impredicative, i.e., cannot be the ML-fragment, since type schemes (rank 1 types) can be substituted.

We next give a reduction from typability to type checking by the use of [ ]-applications.

Proposition $5 \exists \sigma . \Gamma \vdash_{p} P: \sigma$ if and only if $\Gamma, x: \forall t_{1} .\left(t_{1} \rightarrow t_{2}\right) \vdash_{p} x[] P: t_{2}$.

The problem of typability for [ ]-application terms is reduced to the type checking problem of [ ]-application terms. From Theorem 4] the type checking problem for [ ]-application terms becomes undecidable.

Theorem 5 (no ${ }^{T} \mathbf{5}$ ). The problem of type checking for []-application terms is undecidable.

Remarks 7 (no 1 ) From Remark 6, the type checking problem for [ ]-application terms becomes undecidable at rank 3.

Remarks 8 (no(nf) ${ }^{\text {8, }}$ ) From Remark 4 and Proposition 5 the type checking problem for $\mathrm{DF} \&[]$ terms is undecidable at rank 3 , even when the given preterm is a normal form.

\section{Related Work and Concluding Remarks}

\subsection{Related Work and Summary of Results}

Relating to Proposition 2, the problem of strong type inference is also undecidable for domain-free ML with non-sorted variables 4], since the given proof with a slight modification still works for the definition of $T$, where type variable $t$ is replaced with variable $x$, and

$$
\tau::=x \mid \tau \rightarrow \tau
$$

by the use of a single syntactic category of variables $x$. This implies that strong type inference is undecidable for domain-free $\lambda 2$ with non-sorted variables.

Our result also implies a negativ 2 answer to the question posed by Barthe and Sørensen [4] to know whether the problem of type checking is decidable for

${ }^{2}$ Following [5], type checking of domain-free $\lambda 2$ becomes decidable when the given term is in $\beta$-normal form. By the use of domain-erasing translation from Churchstyle to domain-free $\lambda 2$ inducing $\beta$-redexes, Barthe and Sørensen [5] proved that the type inference problem is undecidable for domain-free $\lambda 2$ with sorted variables. Although both works are based on [28, the distinction is that our reduction induces no redex, see Remark 1, and our method with a slight modification is also applicable to the strong type inference problem of domain-free $\lambda 2$ with non-sorted variables. 
domain-free $\lambda 2$ and $\lambda \omega$. Moreover, the type checking problem for domain-free $\lambda \mu$-calculus introduced in [10] also becomes undecidable.

Besides already known ones, we summarize results obtained here, written in italic type marked with superscript. "no (nf)" means that the problem is undecidable even if the given term is a normal form, and "(str)" denotes the strong type inference problem. "yes (nf)" means that the problem becomes decidable only if the given term is a normal form.

For positive results, it is noted that Odersky and Läufer 22 have proposed a decidable extension of ML with fully explicit type scheme annotations so that the system can express polymorphic function arguments. See also Garrigue and Rémy [13] for a conservative extension of ML with semi-explicit first-class polymorphism.

Decidability of type inference, typability, and type checking for $\lambda 2$

\begin{tabular}{|c|c|c|c|c|c|}
\hline$\lambda 2$ & Church & Domain-Free & ]-App. & DF\&[ & Curry \\
\hline$? \vdash P: ?$ & no(nf) 28 & $n o(n f, s t r)^{P 2}$ & $n o^{T 3}$ & $n o(n f, s t r){ }^{n 2}$ & \\
\hline$\Gamma \vdash P: ?$ & yes 31 & $n o(n f)^{72}$ & $n o^{T 4}$ & $n o(n f)^{H 4}$ & \\
\hline$\Gamma \vdash P: \sigma ?$ & yes 31 & $\operatorname{yes}(\mathrm{nf})^{[5]}, n o^{T 2}$ & $n o^{75}$ & $n o(n f)^{18}$ & no 32 \\
\hline
\end{tabular}

\section{Decidability of type inference}

\begin{tabular}{|c|c|c|c|c|c|}
\hline$? \vdash P: ?$ & Church & Domain-Free & -App. & DF\&[ & Curry \\
\hline$\lambda 2$ & no(nf) & $n o(n f, s t r)$ & $n o^{13}$ & $n o(n f, s t r)$ & no 32 \\
\hline rank 2 & no(nf) & $n o(n f, s t r)$ & no & $n o(n f, s t r)$ & yes(str) 17 \\
\hline ML & no(nf) & $n o(n f, s t r)^{f} 2$ & $\mathrm{no}^{\mathrm{H} 5}$ & $n o(n f, s t r)+2$ & yes 18 \\
\hline
\end{tabular}

yes(str) ${ }^{[17]}$ and yes ${ }^{17}$ below provided that the given context assigns closed type schemes to object variables.

\section{Decidability of typability}

\begin{tabular}{|c|c|c|c|c|c|}
\hline$\Gamma \vdash P: ?$ & Church & Domain-Free & -App. & DF\& & Curry \\
\hline$\lambda 2$ & yes 31 & $n o(n f)^{72}$ & $n o^{T 4}$ & $n o(n f)$ & no 32 \\
\hline rank 2 & yes & $n o(n f)^{d 1}$ & $n o^{h[6]}$ & $n o(n f)^{f \sqrt{4}}$ & yes 17 \\
\hline ML & yes & yes & yes & yes & yes \\
\hline
\end{tabular}

The well-known $\mathcal{W}$ [219] is also applicable to the typability for ML in domainfree, [ ]-applications, and DF\&[ ] cases. Remarked that typable terms at rank 1 coincide with typable terms in ML-fragment, and that rank 1 types are applied to type-directed compilation [23]. 


\section{Decidability of type checking}

\begin{tabular}{|c|c|c|c|c|c|}
\hline$\Gamma \vdash P: \sigma ?$ & Church & Domain-Free & ]-App. & DF\&[ & Curry \\
\hline$\lambda 2$ & yes 31 & $\operatorname{yes}(\mathrm{nf})^{5}, n o^{1 / 2}$ & $n o^{T[5}$ & $n o(n f)$ & no \\
\hline rank 3 & yes & yes(nf), no & $n o^{h[7]}$ & $n o(n f)^{n}[\underline{8}$ & no 32 \\
\hline rank 2 & yes & yes(nf),? & $?$ & $?$ & yes $[19]$ \\
\hline ML & yes & yes & yes & yes & yes \\
\hline
\end{tabular}

\subsection{Final Observation and Concluding Remarks}

It is worthwhile to mention the striking contrast between yes $\frac{18}{18}$, yes(str) $\frac{17}{17}$ and no(nf) $)^{[28}, n o(n f, s t r)^{H[2}, n o^{h[5}, n o(n f, s t r)^{h 2}$. Pfenning [26] has proved that the partial type reconstruction problem is undecidable for a predicative fragment of $\lambda 2$. However, the undecidability has been explicitly discussed neither from the viewpoint of rank nor type information of domains and holes [27. Our result shows that existence of type information makes type inference problems undecidable for ML-fragment, in contrast with the case of Curry-style [18]. From this, the partial type reconstruction problem becomes undecidable for the rank 2 fragment of $\lambda 2$, and moreover making polymorphic domains free and the use of type-holes [ ] are independently responsible for the undecidability. The distinction between yes and no for ML-fragment can be observed in the following.

Given a term $M$ in Church-style ML. Then consider a problem to find a context and a type such that ? $\vdash M:$ ? holds in Church-style ML. First consider the typability problem of $\||M|\|$ in Curry-style ML. Let $\left\{x_{1}, \cdots, x_{m}\right\}$ be a set of free variables in $M$. Let $\Gamma$ be $x_{1}: \forall t . t, \cdots, x_{m}: \forall t . t$. If $\||M|\|$ is typable, then we have the principal type $\tau$ of $\||M|\|$, such that $\mathcal{W}(\Gamma,\||M|\|)=(S, \tau)$ and $\Gamma \vdash\||M|\|: \tau$ in Curry-style ML. If $\||M|\|$ is not typable, then $M$ is not typable in Church-style, see (1) and (2) in Section 3.

Assume that $\||M|\|$ is typable. Let $x$ be a free variable appeared $k$ times in $M(k \geq 1)$. From the derivation of $\Gamma \vdash\||M|\|: \tau$ in Curry-style ML, we obtain principal monotypes for each occurrence of $x$ in $\||M|\|$, say $x: \tau_{1}, \cdots, x: \tau_{k}$. Without loss of generality, assume that $x$ appear as one of the following forms in $M$ :

(1) $x$ is used polymorphically in $M$, such that

$$
\left\{\begin{array}{c}
x\left[\tau_{11}\right]\left[\tau_{12}\right] \cdots\left[\tau_{1 n}\right] \\
\cdots \cdots \\
x\left[\tau_{k 1}\right]\left[\tau_{k 2}\right] \cdots\left[\tau_{k n}\right]
\end{array}\right.
$$

(2) $k$ occurrences of $x$ have no type applications in $M$.

In order to solve the original type inference problem $(? \vdash M$ ? in Church-style ML), we have to check that type of the given term $M$ can be the same as the inferred type $\tau$ of the erasure, see also (-1) and (-2) in Section 3, and then at least 
we have to find an answer to the following second-order unification problem: Case of (1)

$$
\left\{\begin{array}{c}
F \tau_{11} \tau_{12} \cdots \tau_{1 n} \doteq \tau_{1} \\
\cdots \cdots \\
F \tau_{k 1} \tau_{k 2} \cdots \tau_{k n} \doteq \tau_{k}
\end{array}\right.
$$

where $F$ is an unknown second-order variable with $n$-arity, in which every argument of $F$ contains no variables for the unification problem. Type variables in $\tau_{1}, \cdots, \tau_{k}$ are used as unknown first-order variables for the unification. This unification problem is a variant of the problem in Section 5, i.e., the simple instances of the second-order unification problem [28,29] with first-order variables. The case of (2) gives the first-order unification problem:

$\tau_{1} \doteq \tau_{2} \doteq \cdots \doteq \tau_{k}$

Following the observation above, $\mathcal{W}$ plus an answer to the second-order unification problem could solve the problems of type inference in the cases of Church, domain-free, [ ]-applications, and DF\&[ ], i.e., additional type information gives more constraints to be solved.

Another observation is about the definition of preterms. Alternatively, one can define preterms with full annotations, such that $Q::=x\left|\lambda x:[\sigma]^{a} . Q\right| Q Q|\lambda t . Q| Q[\sigma]^{a}\left|\lambda x:[]^{a} . Q\right| Q[]^{a}$

Then $\Gamma \vdash_{p} Q: \sigma ; \Delta$ can be similarly defined, and the same results in 7.1 also hold for the preterms following a similar pattern.

\section{Acknowledgements}

We are grateful to J. Roger Hindley and Horai-Takahashi Masako for helpful discussions. We would like to thank Frank Pfenning for valuable comments on this work, and Gilles Barthe and Morten Heine B. Sørensen for pointing out their paper in the revised version [5]. The authors are also grateful to anonymous referees for their constructive comments and suggestions.

\section{References}

1. Barendregt, H. P.: Lambda Calculi with Types, Handbook of Logic in Computer Science Vol.II, Oxford University Press, pp. 1-189, 1992.

2. Boehm, Hans-J.: Partial Polymorphic Type Inference is Undecidable, Proc. 26th Annual Symposium of Fundations of Computer Science, pp. 339-345, 1985.

3. Barthe, G., Hatcliff, J., and Sørensen, M. H.: CSP Translations and Applications: The Cube and Beyond, Proc. ACM SIGPLAN on Continuations, pp. 1-31, 1996.

4. Barthe, G. and Sørensen, M. H.: Domain-Free Pure Type Systems, Lecture Notes in Computer Science 1234, pp. 9-20, 1997.

5. Barthe, G. and Sørensen, M. H.: Domain-Free Pure Type Systems.

6. Church, A.: A Formulation of the Simple Theory of Types, The Journal of Symbolic Logic, Vol. 5, pp. 56-68, 1940.

7. Curry, H. B.: Functionality in combinatory logic, Proc. National Academy of Sciences of the USA 20, pp. 584-590, 1934. 
8. Curry, H. B., Feys, R. and Craig, W.: Combinatory Logic, Volume 1 (Third printing), North-Holland, 1974.

9. Damas, L. and Milner, R.: Principal type-schemes for functional programs, Proc. ACM Symposium on Principles of Programming Languages, pp. 207-212, 1982.

10. Fujita, K.: Explicitly Typed $\lambda \mu$-Calculus for Polymorphism and Call-by-Value, Lecture Notes in Computer Science 1581, pp. 162-176, 1999.

11. Fujita, K.: Type Inference for Domain-Free $\lambda 2$, Technical Report in Computer Science and Systems Engineering CSSE-5, Kyushu Institute of Technology, 1999.

12. Fujita, K: Undecidability of Partial Type Reconstruction, Computer Software, Vol. 17, No. 2, pp. 40-44, 2000 (in Japanese).

13. Garrigue, J. and Rémy, D.: Semi-Explicit First-Class Polymorphism for ML, Information and Computation, 1999.

14. Harper, R. and Lillibridge, M.: ML with callcc is unsound, The Types Form, 8 July 1991.

15. Harper, R. and Mitchell, J. C.: On The Type Structure of Standard ML, ACM Trans. on Programming Languages and Systems, Vol. 15, No. 2, pp. 210-252, 1993.

16. Hindley, J. R.: Basic Simple Type Theory, Cambridge University Press, 1997.

17. Kfoury, A. J. and Tiuryn, J. : Type Reconstruction in Finite Rank Fragments of the Second-Order $\lambda$-Calculus, Information and Computation 98, pp. 228-257, 1992.

18. Kfoury, A. J., Tiuryn, J. and Urzyczyn, P.: An Analysis of ML Typability, Journal of the Association for Computing Machinery, Vol. 41, No. 2, pp. 368-398, 1994.

19. Kfoury, A. J. and Wells, J. B.: A direct algorithm for type inference in the rank-2 fragment of the second-order $\lambda$-calculus, Proc. ACM LISP and Functional Programming, pp. 196-207, 1994.

20. Leivant, D.: Finitely Stratified Polymorphism, Information and Computation 93, pp. 93-113, 1991.

21. Milner, R.: A Theory of Type Polymorphism in Programming, Journal of Computer and System Sciences 17, pp. 348-375, 1978.

22. Odersky, M. and Läufer, K.: Putting Type Annotations to Work, Proc. ACM Symposium on Principles of Programming Languages, pp. 54-76, 1996.

23. Ohori, A. and Yoshida, N.: Type Inference with Rank 1 Polymorphism for TypeDirected Compilation for ML, Proc. ACM Conference on Functional Programming, pp. 160-171, 1999.

24. Parigot, M.: $\lambda \mu$-Calculus: An Algorithmic Interpretation of Classical Natural Deduction, Lecture Notes in Computer Science 624, pp. 190-201, 1992.

25. Pfenning, F.: Partial polymorphic type inference and higher-order unification, Proc. ACM Conference on Lisp and Functional Programming, pp. 153-163, 1998.

26. Pfenning, F.: On the undecidability of partial polymorphic type reconstruction, Fundamenta Informaticae 19, pp. 185-199, 1993.

27. Pfenning, F.: private communication, 9 June 1999.

28. Schubert, A.: Second-order unification and type inference for Church-style, Tech. Report TR 97-02 (239), Institute of Informatics, Warsaw University, January 1997.

29. Schubert, A.: Second-order unification and type inference for Church-style, Proc. ACM Symposium on Principles of Programming Languages, pp. 279-288, 1998.

30. Tiuryn, J.: Type Inference Problems: A Survey, Lecture Notes in Computer Science 452, pp. 105-120, 1990.

31. Van Benthem Jutting, L. S.: Typing in Pure Type Systems, Information and Computation 105, pp. 30-41, 1993.

32. Wells, J. B.: Typability and Type Checking in the Second-Order $\lambda$-Calculus Are Equivalent and Undecidable, Proc. IEEE Symposium on Logic in Computer Science, pp. 176-185, 1994. 